\title{
A Particle Filter to Track Multiple Objects
}

\author{
Carine Hue Jean-Pierre Le Cadre \\ IRISA \\ Campus de Beaulieu \\ 35042 Rennes Cedex, France \\ chue@irisa.fr lecadre@irisa.fr
}

\author{
Patrick Pérez \\ Microsoft Research \\ St George House, 1 Guildhall Street \\ Cambridge, CB2 3NH, UK \\ pperez@microsoft.com
}

\begin{abstract}
We address the problem of tracking multiple objects encountered in many situations in signal or image processing. We consider stochastic dynamic systems nonlinearly and uncompletely observed. The difficulty lies on the fact that the estimation of the states requires the assignation of the observations to the multiple targets. We propose an extension of the classical particle filter where the stochastic vector of assignation is estimated by a Gibbs sampler. The merit of the method is assessed in bearings-only context and we present one application in image-based tracking.
\end{abstract}

\section{Introduction}

Multiple object tracking (MTT) deals with state estimation of an unknown number of moving targets. Available measurements may both arise from the targets if they are detected, and from clutter. Clutter is generally considered as a model describing false alarms. Its (spatio-temporal) statistical properties are quite different from target ones, which makes possible the extraction of target tracks from clutter. To perform multiple object tracking the observer has at his disposal a huge amount of data, possibly collected on multiple receivers. In signal processing, elementary measurements are receiver outputs, e.g., bearings, ranges, timedelays, Dopplers, etc. In image-based tracking they have to be computed from the images.

But the main difficulty comes from the assignment of a given measurement to a target model. These assignments are generally unknown, as are the true target models. This is a neat departure from classical estimation problems. Thus, two distinct problems have to be solved jointly: data association and estimation. As long as the association is considered in a deterministic way, the hypothesis associations must be exhaustively enumerated, which leads to a NPhard problem (as in JPDAF and MHT algorithms [3] for instance). As soon as the association variables are consid- ered as stochastic variables and moreover statistically independent like in the Probabilistic MHT (PMHT), the complexity is reduced. However, the above algorithms do not cope with non linear state or measurement models and non Gaussian state or measurement noises. Under such assumptions (stochastic state equation and non linear state or measurement equation, non Gaussian noises), particle filters are particularly adapted. They mainly consist in propagating a weighted set of particles which approximates the probability density of the state conditionally to the observations. Particle filtering can be applied under very weak hypotheses, is able to cope with heavy clutter, and is very easy to implement. Numerous versions have been used in various contexts: the bootstrap filter for target tracking in [4], the Condensation algorithm in image analysis [5] are two examples among others. In image analysis a probabilistic exclusion principle has been developed in [7] to track multiple objects but the algorithm is very dependent on the observation model and seems costly to extend for more than two objects. We propose here a quite general algorithm for multiple object tracking applicable both in signal and image analysis.

This work is organised as follows. In section II, we briefly recall the basic particle filter. Section III deals with our extension of the basic filter to multiple objects. Section IV begins with a validation of our algorithm in the passive sonar context, i.e. to estimate the trajectories of multiple "small" targets from their noisy bearings. Then it is used to track pedestrians in a video-sequence.

\section{The basic particle filter}

For the sake of completeness, the basic particle filter is now briefly reviewed. We consider a dynamic system represented by the stochastic process $\left(X_{t}\right) \in \mathbb{R}^{n_{x}}$ whose temporal evolution is given by the state equation:

$$
X_{t}=F_{t}\left(X_{t-1}, V_{t}\right) .
$$


- Initialization: $\left\{\begin{array}{l}s_{0}^{n} \sim p\left(X_{0}\right) \\ q_{0}^{n}=1 / N\end{array} \quad n=1, \ldots, N\right.$.

- For $t=1, \ldots, T$ :

○ Proposal: sample $\tilde{s}_{t}^{n}$ from $f\left(X_{t} \mid X_{t-1}=s_{t-1}^{n}, Y_{t}=y_{t}\right)$ for $n=1, \ldots, N$.

○Weighting: $\left\{\begin{array}{l}\text { Compute un-normalised weights: } \tilde{q}_{t}^{n}=q_{t-1}^{n} \frac{p\left(\tilde{s}_{t}^{n} \mid s_{t-1}^{n}\right) l_{t}\left(y_{t} ; \tilde{s}_{t}^{n}\right)}{f\left(\tilde{s}_{t}^{n} \mid s_{t-1}^{n}, y_{t}\right)} \text { for } n=1, \ldots, N . \\ \text { Normalise weights: } q_{t}^{n}=\frac{\tilde{q}_{t}^{n}}{\sum_{n=1}^{N} \tilde{q}_{t}^{n}} \text { for } n=1, \ldots, N .\end{array}\right.$

○ Return $\widehat{\mathbb{E}} g\left(X_{t}\right)=\sum_{n=1}^{N} q_{t}^{n} g\left(\tilde{s}_{t}^{n}\right)$.

○ Calculate $\hat{N}_{e f f}=\frac{1}{\sum_{n=1}^{N}\left(q_{t}^{n}\right)^{2}}$.

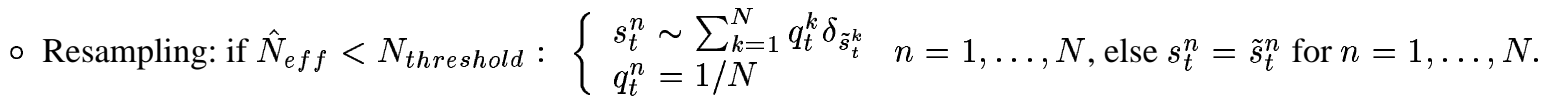

Figure 1. Basic particle filter with adaptive resampling .

It is observed at discrete times via realizations of the stochastic process $\left(Y_{t}\right) \in \mathbb{R}^{n_{y}}$ governed by the measurement model:

$$
Y_{t}=H_{t}\left(X_{t}, W_{t}\right) .
$$

The two processes $\left(V_{t}\right) \in \mathbb{R}^{n_{v}}$ and $\left(W_{t}\right) \in \mathbb{R}^{n_{w}}$ in (1) and (2) are only supposed to be independent white noises. Moreover, it is to be noted that no linearity hypothesis on $F_{t}$ and $H_{t}$ is done. We will denote by $Y_{0: t}$ the sequence of the random variables $\left(Y_{0}, \ldots, Y_{t}\right)$ and by $y_{0: t}$ one realization of this sequence.

Our problem consists in computing at each time $t$ the conditional density $L_{t}$ of the state $X_{t}$ given all the observations accumulated up to $t$, i.e., $L_{t}=p\left(X_{t} \mid Y_{0}=y_{0}, \ldots, Y_{t}=y_{t}\right)$ and also in estimating any functional of the state $g\left(X_{t}\right)$ by the expectation $\mathbb{E}\left(g\left(X_{t}\right) \mid Y_{0: t}\right)$. The Recursive Bayesian filter, also named Optimal Filter, resolves exactly this problem in two steps at each time $t$ [4].

Suppose we know $L_{t-1}$. The prediction step is done according to the following equation:

$$
\begin{aligned}
& p\left(X_{t}=x_{t} \mid Y_{0: t-1}=y_{0: t-1}\right)= \\
& \int_{\mathbb{R}^{n_{x}}} p\left(X_{t}=x_{t} \mid X_{t-1}=x\right) L_{t-1}(x) d x .
\end{aligned}
$$

The observation $y_{t}$ enables us to correct this prediction using the Bayes's rule:

$$
L_{t}\left(x_{t}\right)=\frac{p\left(Y_{t}=y_{t} \mid x_{t}\right) p\left(X_{t}=x_{t} \mid y_{0: t-1}\right)}{\int_{\mathbb{R}^{n_{x}}} p\left(Y_{t}=y_{t} \mid x\right) p\left(X_{t}=x \mid y_{0: t-1}\right) d x} .
$$

These equations provide a closed-form recursion if we assume restrictive hypothesis such as Kalman Filter's ones. The functions $F_{t}$ and $G_{t}$ are then supposed to be linear and the noises $V_{t}$ and $W_{t}$ to be Gaussian. Unfortunately this modelling is not appropriate in many problems in signal and image processing, which renders the calculations of the integrals in (3) and (4) infeasible (no closed-form).

The original particle filter, named bootstrap filter [4], proposes to approximate the densities $\left(L_{t}\right)_{t}$ by a finite weighted sum of $N$ Dirac densities centred on elements of $\mathbb{R}^{n_{x}}$, named particles. The application of the bootstrap filter requires that one knows how:

- to sample from initial prior marginal $p\left(X_{0}\right)$;

- to sample from $p\left(V_{t}\right)$ for all $t$;

- to compute $p\left(Y_{t}=y_{t} \mid X_{t}=x_{t}\right)$ for all $t$ through a known function $l_{t}$ such that $l_{t}(y ; x) \propto$ $p\left(Y_{t}=y \mid X_{t}=x\right)$ where missing normalization must not depend on $x$.

The algorithm then consists in making evolve the particle set $S_{t}=\left(s_{t}^{n}, q_{t}^{n}\right)_{n=1, \ldots, N}$, where $s_{t}$ is the particle position and $q_{t}$ its weight, and to use it to estimate the density $L_{t}$ by the density $L_{S_{t}}=\sum_{n=1}^{N} q_{t}^{n} \delta_{s_{t}^{n}}$. The weak convergence of the probability density $L_{S_{t}}$ towards $L_{t}$ when $N \rightarrow \infty$ with rate $1 / \sqrt{N}$ can be proved. To avoid the degeneracy of the particle set, i.e. only few particles with high weights and the others with very small ones, a resampling is done in an adaptive way when the number of effective particles, estimated by $\hat{N}_{e f f}$, is under a given threshold [2]. Besides the discretization of the filtering integrals, the use of such particles enables to voice many hypothesis on the position of the object and to keep in the long term only the particles whose position is likely given the sequence of observations. In bootstap filter, the particles are "moved" by sampling from the dynamics (1), and importance sampling theory shows that the weighting is only based on likelihood evaluations. In the most general setting [2], the displacement of particles is obtained by sampling from an appropriate density 
$f$ which might depend on the data as well. The complete procedure is summarized in figure 1 . The reader will find more details on the different filters in [4], [5] or [2] and on adaptive resampling in [6] and [2]. After these recalls, we propose an extension of this algorithm to multiple-object tracking.

\section{Multiple object particle filters}

\subsection{Notations}

Let $M$ be the number of objects to track (first assumed to be known and fixed). The state vector we have to estimate is made by concatenating the state vector of each object. At time $t, X_{t}=\left(X_{t}^{1}, \ldots, X_{t}^{M}\right)$ follows the state equation (1) decomposed in $M$ partial equations:

$$
X_{t}^{i}=F_{t}^{i}\left(X_{t-1}^{i}, V_{t}^{i}\right) \quad \forall i=1, \ldots, M .
$$

The noises $\left(V_{t}^{i}\right)$ and $\left(V_{t}^{i^{\prime}}\right)$ are only supposed to be white both temporally and spatially, independent for $i \neq i^{\prime}$. The observation vector at time $t$ is denoted by $y_{t}=$ $\left(y_{t}^{1}, \ldots, y_{t}^{m_{t}}\right)$. Following the seminal ideas of R. Streit and T. Luginbuhl [10], we introduce the stochastic vector $K_{t} \in\{1, \ldots, M\}^{m_{t}}$ such that $K_{t}^{j}=i$ if $y_{t}^{j}$ is issued from the $i^{t h}$ object. In this case, $y_{t}^{j}$ is a realization of the stochastic process:

$$
Y_{t}^{j}=H_{t}^{i}\left(X_{t}^{i}, W_{t}^{j}\right) \text { if } K_{t}^{j}=i .
$$

Again, the noises $\left(W_{t}^{j}\right)$ and $\left(W_{t}^{j^{\prime}}\right)$ are only supposed to be white noises, independent for $j \neq j^{\prime}$. We assume that the functions $H_{t}^{i}$ are such that they can be associated to functional forms $l_{t}^{i}$ defined by $l_{t}^{i}(y ; x) \propto p\left(Y_{t}^{j}=y \mid K_{t}^{j}=i, X_{t}^{i}=x\right)$.

We make the assumption that one measurement can originate from one object or from the clutter and that one object can produce zero or several measurements at one time. For that, we dedicate the model 0 to false alarms. The false alarms are supposed to be uniformly distributed in the observation area. Their number is assumed to arise from a Poisson density of parameter $\lambda V$ where $V$ is the volume of the surveillance area and $\lambda$ the number of false alarms by volume unity. Of course, we do not associate any kinematic model to false alarms and then no particles represent their density. Let $\pi_{t} \in[0,1]^{M+1}$ defined by $\pi_{t}^{i}=\mathbb{P}\left(K_{t}^{j}=i\right)$ for all $j=1, \ldots m_{t}$. This definition implicitly assumes that the probabilities $\pi_{t}^{i}$ are independent of the measurements as their indexation is arbitrary. These assumptions imply that $m_{t}$ may differ from $M$ and that the association is exclusive and exhaustive. In particular, $\sum_{i=0}^{M} \pi_{t}^{i}=1$. Furthermore, it is assumed that the assignment vector $K_{t}$ has independent components (see[10]). To estimate the density $L_{t}=p\left(X_{t}=\left(X_{t}^{1}, \ldots, X_{t}^{M}\right) \mid y_{0: t}\right)$, we propose to use particles whose dimension is the sum of the ones of the individual state spaces corresponding to each object, as in [7]. Each of these concatenated vectors then gives jointly a representation of all objects. Let us present the proposed multiple object particle filter (MOPF).

\subsection{The MOPF}

The initial particle set $S_{0}=\left(s_{0}^{n}, 1 / N\right)_{n=1, \ldots, N}$ is such that each component $s_{0}^{n, i}$ for $i=1, \ldots, M$ is sampled from $p\left(X_{0}^{i}\right)$ independently from the others. Assume we have obtained $S_{t-1}=\left(s_{t-1}^{n}, q_{t-1}^{n}\right)_{n=1, \ldots, N}$ with $\sum_{n=1}^{N} q_{t-1}^{n}=1$. Each particle is a vector of dimension $\sum_{i=1}^{M} n_{x}^{i}$ where we denote by $s_{t-1}^{n, i}$ the $i^{t h}$ component of $s_{t-1}^{n}$ and where $n_{x}^{i}$ designates the dimension of object $i$.

The prediction is performed by sampling from some proposal density $f$. In bootstrap filter case, $f$ coincides with the dynamics (5):

$$
\text { For } n=1, \ldots, N \tilde{s}_{t}^{n}=\left(\begin{array}{c}
F_{t}^{1}\left(s_{t-1}^{n, 1}, v_{t}^{n, 1}\right) \\
\vdots \\
F_{t}^{M}\left(s_{t-1}^{n, M}, v_{t}^{n, M}\right)
\end{array}\right)
$$

with $\left(v_{t}^{n, i}\right)$ being realizations of $\left(V_{t}^{i}\right)$. Examine now the computation of the likelihood of the observations conditioned by the $n^{t h}$ particle. We can write for all $n=$ $1, \ldots, N$ :

$$
\begin{aligned}
& p\left(Y_{t}=\left(y_{t}^{1}, \ldots, y_{t}^{m_{t}}\right) \mid X_{t}=\tilde{s}_{t}^{n}\right)=\prod_{j=1}^{m_{t}} p\left(y_{t}^{j} \mid \tilde{s}_{t}^{n}\right) \\
& \propto \prod_{j=1}^{m_{t}}\left[\frac{\pi_{t}^{0}}{V}+\sum_{i=1}^{M} l_{t}^{i}\left(y_{t}^{j} ; \tilde{s}_{t}^{n, i}\right) \pi_{t}^{i}\right] .
\end{aligned}
$$

It must be noted that first equality in (8) is true only under the assumption of conditional independence of the measures, which we will make. Moreover, the normalization factors between $l_{t}^{i}$ and $p\left(Y_{t}^{j}=y \mid K_{t}^{j}=i, X_{t}^{i}=x\right)$ must be the same for all $i$ to write the second equality in (8).

It remains to estimate the association probabilities $\left(\pi_{t}^{i}\right)_{i=1, \ldots, M}$ which can be seen as the stochastic coefficients of the $M$-component mixture. To estimate them we propose to use a Gibbs sampler whose principles are briefly recalled (see [1] or [9] for more details). For $\theta=\left(X_{t}, K_{t}, \Pi_{t}\right)$, it consists in generating a Markov chain which converges to the stationary distribution $p\left(\theta \mid Y_{0: t}\right)$ which cannot be sampled directly. Given a partition $\theta^{1}, \ldots, \theta^{P}$ of $\theta$, one samples alternatively from the conditional posterior distribution of each component of the partition. Assume the $\tau$ first elements of the Markov chain $\left(\theta_{1}, \ldots, \theta_{\tau}\right)$ have been drawn. We sample the $P$ components of $\theta_{\tau+1}$ as follows:

$$
\begin{array}{lll}
\text { Draw } & \theta_{\tau+1}^{1} & \text { from } p\left(\theta^{1} \mid Y_{0: t}, \theta_{\tau}^{2}, \ldots, \theta_{\tau}^{P}\right) \\
\text { Draw } \theta_{\tau+1}^{2} & \text { from } p\left(\theta^{2} \mid Y_{0: t}, \theta_{\tau+1}^{1}, \theta_{\tau}^{3}, \ldots, \theta_{\tau}^{P}\right)
\end{array}
$$


- Initialization: $\left\{\begin{array}{l}s_{0}^{n} \sim p\left(X_{0}\right) \\ q_{0}^{n}=1 / N\end{array} \quad n=1, \ldots, N\right.$.

- For $t=1, \ldots, T$ :

○ Proposal: sample $\tilde{s}_{t}^{n}$ from $f\left(X_{t} \mid X_{t-1}=s_{t-1}^{n}, Y_{t}=y_{t}\right)$ for $n=1, \ldots, N$.

- Weighting:

1. Initialization of the Gibbs sampler: $\begin{cases}\pi_{t, 0}^{i}=\frac{1-\pi_{t}^{0}}{M} & i=1, \ldots, M ; \\ X_{t, 0}^{i}=\sum_{n=1}^{N} q_{t-1}^{n} \tilde{s}_{t}^{n, i} & i=1, \ldots, M .\end{cases}$

2. For $\tau=0, \ldots, \tau_{\text {end }}$ :

a. $K_{t, \tau+1}^{j} \sim p\left(K_{t, \tau+1}^{j}=i\right) \propto \begin{cases}\pi_{t, \tau}^{i} l_{t}^{i}\left(y_{t}^{j} ; x_{t, \tau}^{i}\right) & \text { if } i=1, \ldots, M ; \\ \pi_{t}^{0} / V & \text { if } i=0 .\end{cases}$

b. $\pi_{t, \tau+1}^{1: M} \sim \mathcal{D}\left(\left(1+n^{i}\left(K_{t, \tau+1}\right)\right)_{i=1, \ldots, M}\right), n^{i}(K) \triangleq \sharp\left\{j: K^{j}=i\right\}$.

c. For each $i$ such that $\exists j^{1}, \ldots, j^{i} / K_{t, \tau+1}^{j^{l}}=i$,

$$
\begin{aligned}
& \text {-\{ }\left\{\begin{array}{l}
\sigma_{\tau+1}^{n}=\tilde{s}_{t}^{n, i} \\
\chi_{\tau+1}^{n}=\frac{p\left(y_{t}^{j^{1}}, \ldots, y_{t}^{j^{i}} \mid X_{t}^{i}=\sigma_{\tau+1}^{n}\right) q_{t-1}^{n}}{\sum_{n=1}^{N} p\left(y_{t}^{j^{1}}, \ldots, y_{t}^{j^{i}} \mid X_{t}^{i}=\sigma_{\tau+1}^{n}\right) q_{t-1}^{n}}
\end{array} \quad n=1, \ldots, N .\right. \\
& \text { - } X_{t, \tau+1}^{i} \sim \sum_{n=1}^{N} \chi_{\tau+1}^{n} \delta_{\sigma_{\tau+1}^{n}}
\end{aligned}
$$

$d$. For each $i$ such that $\nexists j / K_{t}^{j}=i, X_{t, \tau+1}^{i} \sim \sum_{n=1}^{N} q_{t-1}^{n} \delta_{\tilde{s}_{t}^{n, i}}$.

3. $\hat{\pi}_{t}^{i}=\frac{1}{\tau_{\text {beg }}-\tau_{\text {end }}} \sum_{\tau=\tau_{\text {beg }}}^{\tau_{\text {end }}} \pi_{t, \tau}^{i} \quad i=1, \ldots, M$.

4. $l_{t}\left(Y_{t}=\left(y_{t}^{1}, \ldots, y_{t}^{m_{t}}\right) \mid \tilde{s}_{t}^{n}\right)=\prod_{j=1}^{m_{t}}\left[\frac{\pi_{t}^{0}}{V}+\sum_{i=1}^{M} l_{t}^{i}\left(y_{t}^{j} ; \tilde{s}_{t}^{n, i}\right) \hat{\pi}_{t}^{i}\right] n=1, \ldots, N$.

5. $q_{t}^{n} \propto q_{t-1}^{n} \frac{\left.p\left(\tilde{s}_{t}^{n}\right) \mid s_{t-1}^{n}\right) l_{t}\left(Y_{t} \mid \tilde{s}_{t}^{n}\right)}{f\left(\tilde{s}_{t} \mid s_{t-1}, y_{t}\right)} \quad n=1, \ldots, N$.

○ Return $\widehat{\mathbb{E}} g\left(X_{t}\right)=\sum_{n=1}^{N} q_{t}^{n} g\left(\tilde{s}_{t}^{n}\right)$.

○ Calculate $\hat{N}_{e f f}=\frac{1}{\sum_{n=1}^{N}\left(q_{t}^{n}\right)^{2}}$.

$\circ$ Resampling: if $\hat{N}_{\text {eff }}<N_{\text {threshold }}:\left\{\begin{array}{l}s_{t}^{n} \sim \sum_{k=1}^{N} q_{t}^{k} \delta_{\tilde{s}_{t}^{k}} \quad n=1, \ldots, N \text {, else } s_{t}^{n}=\tilde{s}_{t}^{n} \text { for } n=1, \ldots, N \\ q_{t}^{n}=1 / N\end{array}\right.$

Figure 2. MOPF: multiple object particle filter with adaptive resampling.

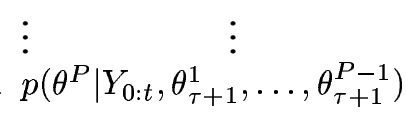

In our case, at a given instant $t$, the partitionning of $\theta$ is:

$$
\begin{cases}\theta^{j}=K_{t}^{j} & \text { for } j=1, \ldots, m_{t} \\ \theta^{m_{t}+i}=\pi_{t}^{i} & \text { for } i=1, \ldots, M \\ \theta^{m_{t}+M+i}=X_{t} & \text { for } i=1, \ldots, M\end{cases}
$$

We now detail the different steps of this Gibbs sampler. 1. The initialization of Gibbs sampler consists in assigning uniform association probabilities, i.e., $\pi_{t, 0}^{i}=\frac{1-\pi_{0}}{M}$ for all $i=1, \ldots, M$, and taking $X_{t, 0}=\sum_{n=1}^{N} q_{t-1}^{n} \tilde{s}_{t}^{n}$, i.e., the centroid of the predicted particle set. Then, suppose that at instant $t$ we have already simulated $\left(\theta_{t, 1}, \ldots, \theta_{t, \tau}\right)$.

2 . The $\tau+1^{\text {th }}$ iteration is handled as follows. a. As the $\left(K_{t}^{j}\right)_{j=1, \ldots, m_{t}}$ are supposed to be independent, their individual conditional density reads:

$$
p\left(K_{t}^{j} \mid Y_{0: t}, X_{t},\left(K_{t}^{l}\right)_{l \neq j}, \Pi_{t}\right)=p\left(K_{t}^{j} \mid Y_{t}^{j}, X_{t}, \Pi_{t}\right) .
$$

$\left(K_{t}^{j}\right)$ are discrete variables and we can write:

$$
\begin{aligned}
& \mathbb{P}\left(K_{t}^{j}=i \mid Y_{t}^{j}=y_{t}^{j}, X_{t}, \Pi_{t}\right) \\
& =\frac{p\left(Y_{t}^{j}=y_{t}^{j} \mid K_{t}^{j}=i, X_{t}, \Pi_{t}\right) \mathbb{P}\left(K_{t}^{j}=i \mid X_{t}, \Pi_{t}\right)}{\left.p_{t}^{j}=y_{t}^{j} \mid X_{t}, \Pi_{t}\right)} \\
& \propto \begin{cases}\pi_{t}^{i} l_{t}^{i}\left(y_{t}^{j} ; x_{t}^{i}\right) & \text { if } i=1, \ldots, M ; \\
\pi_{t}^{0} / V & \text { if } i=0 .\end{cases}
\end{aligned}
$$

The realizations $k_{t, \tau+1}^{j}$ of the vector $K_{t, \tau+1}$ are then sampled according to the weights $p_{t, \tau+1}^{j, 0}=\pi_{t}^{0} / V$, and $p_{t, \tau+1}^{j, i}=\pi_{t, \tau}^{i} l_{t}^{i}\left(y_{t}^{j} ; x_{t, \tau}^{i}\right)$ for $i=1, \ldots, M$. 
$b$. Mixture proportion vector $\Pi_{t, \tau+1}^{1: M}$ is drawn from the conditional density:

$$
\begin{aligned}
& p\left(\Pi_{t}^{1: M} \mid K_{t, \tau+1}, X_{t, \tau}, Y_{0: t}\right)= \\
& p\left(\Pi_{t}^{1}, \ldots, \Pi_{t}^{M} \mid K_{t, \tau+1}^{1}, \ldots, K_{t, \tau+1}^{M}, X_{t, \tau}, Y_{0: t}\right) \propto \\
& p\left(K_{t, \tau+1}^{1}, \ldots, K_{t, \tau+1}^{M} \mid \Pi_{t}^{1}, \ldots, \Pi_{t}^{M}\right) p\left(\Pi_{t}^{1}, \ldots, \Pi_{t}^{M}\right) \\
& =\left(1-\pi_{0}\right) \mathcal{D}\left(\Pi_{t} \mid M,\left\{n^{i}\left(K_{t, \tau+1}\right)_{i=1, \ldots, M}\right\}\right)
\end{aligned}
$$

where we denote by $n^{i}(K)$ the number of $k^{j}$ equal to $i$ and $\mathcal{D}$ stands for Dirichlet distribution.

$X_{t, \tau+1}$ has to be sampled according to the density

$p\left(X_{t} \mid Y_{0: t}, K_{t, \tau+1}, \Pi_{t, \tau+1}\right)=\prod_{i=1}^{M} p\left(X_{t}^{i} \mid Y_{0: t}, K_{t, \tau+1}, \Pi_{t, \tau+1}\right)$

The values of $K_{t, \tau+1}$ can imply that one object is associated with zero or several measurements that is why we decompose the preceding product in two products:

$$
\begin{aligned}
& \prod_{i / \exists j^{1}, \ldots, j^{i} / K_{t, \tau+1}^{j}=i} p\left(X_{t}^{i} \mid Y_{0: t-1}, y_{t}^{j^{1}}, \ldots, y_{t}^{j^{i}}, \Pi_{t, \tau+1}\right) \\
& \prod_{i / \forall j K_{t, \tau+1}^{j} \neq i} p\left(X_{t}^{i} \mid Y_{0: t-1}, \Pi_{t, \tau+1}\right)
\end{aligned}
$$

$c$. Let $i$ be an integer in the first product. We can write

$$
\begin{aligned}
& p\left(X_{t}^{i} \mid Y_{0: t-1}, y_{t}^{j^{1}}, \ldots, y_{t}^{j^{i}}, \Pi_{t, \tau+1}\right)= \\
& \frac{p\left(y_{t}^{j^{1}}, \ldots, j_{t}^{j^{i}} \mid X_{t}^{i}\right) p\left(X_{t}^{i} \mid Y_{0: t-1}\right)}{p\left(y_{t}^{j^{1}}, \ldots, y_{t}^{j^{i}} \mid Y_{0: t-1}\right)}
\end{aligned}
$$

We are not able to sample directly from the density $\frac{p\left(y_{t}^{j^{1}}, \ldots, y_{t}^{j^{i}} \mid X_{t}^{i}\right) p\left(X_{t}^{i} \mid Y_{0: t-1}\right)}{p\left(y_{t}^{j^{1}}, \ldots, y_{t}^{j^{i}} \mid Y_{0: t-1}\right)}$, for the same reasons as those exposed in section 2 to justify the use of the particle filter (intractability of the integrals). We propose to build the particle set $\Sigma_{\tau+1}=\left(\sigma_{\tau+1}^{n}, \chi_{\tau+1}^{n}\right)_{n=1, \ldots, N}$ whose weights $\chi_{\tau+1}^{n}$ measure the likelihood of the observations affected by $K_{t, \tau+1}$ to object $X_{t}^{i}$. More precisely, we let:

$$
\left\{\begin{array}{l}
\sigma_{\tau+1}^{n}=\tilde{s}_{t}^{n, i} \\
\chi_{\tau+1}^{n}=\frac{p\left(y_{t}^{j^{1}}, \ldots, y_{t}^{j^{i}} \mid X_{t}^{i}=\sigma_{\tau+1}^{n}\right) q_{t-1}^{n}}{\sum_{n=1}^{N} p\left(y_{t}^{j^{1}}, \ldots, y_{t}^{j^{i}} \mid X_{t}^{i}=\sigma_{\tau+1}^{n}\right) q_{t-1}^{n}} .
\end{array}\right.
$$

The density $\Lambda_{\tau+1}=\sum_{n=1}^{N} \chi_{\tau+1}^{n} \delta_{\sigma_{\tau+1}^{n}}$ converges weakly to the density $p\left(X_{t}^{i} \mid y_{t}^{j^{1}}, \ldots, y_{t}^{j^{i}}, Y_{0: t-1}\right)$. Not being able to sample from this last density, $X_{t, \tau+1}^{i}$ is drawn as a realization from $\Lambda_{\tau+1}$.

$d$. Now let $i$ be an integer in the second product. As we do not have any measure to correct the predicted particles we draw a realization from the density $\sum_{n=1}^{N} q_{t-1}^{n} \delta_{\tilde{s}_{t}^{n}}$ for $X_{t, \tau+1}^{i}$.

3. After a finite number of iterations, we estimate the vector $\pi_{t}$ by the average of its last realizations:

$$
\hat{\pi}_{t}^{i}=\frac{1}{\tau_{b e g}-\tau_{\text {end }}} \sum_{\tau=\tau_{\text {beg }}}^{\tau_{\text {end }}} \pi_{t, \tau}^{i}
$$

Finally the weights are computed according to (8) using the estimation $\hat{\pi}_{t}^{i}$ of $\pi_{t}^{i}$. Figure 2 summarizes the whole procedure.

\section{Application to bearings-only problems}

We first deal with the classical bearings-only problem using synthetic data. The objects are then "pointobjects" in the $x-y$ plane. Their state vector $X_{t}$ represents the coordinates and the velocities in the $x-y$ plane: $X_{t}=\left(x_{t}, y_{t}, v x_{t}, v y_{t}\right)$. The following multitarget scenario has been considered: three targets follow a nearconstant-velocity model defined by (18). The discretized state equation associated with time period $\Delta t$ is:

$$
X_{t+\Delta t}=\left(\begin{array}{ll}
I d_{2} & \Delta t I d_{2} \\
0 & I d_{2}
\end{array}\right) X_{t}+\left(\begin{array}{c}
\frac{\Delta t^{2}}{2} I d_{2} \\
\Delta t I d_{2}
\end{array}\right) V_{t}
$$

where $I d_{2}$ is the identity matrix in dimension 2 and $V_{t}$ is a Gaussian zero-mean vector of covariance matrix $\Sigma_{V}=$ $\left[\begin{array}{ll}\sigma_{x}^{2} & 0 \\ 0 & \sigma_{y}^{2}\end{array}\right]$. Let $\hat{X}_{t}$ be the estimation of $X_{t}$ computed by the MOPF with $g(x)=x$, i.e. , $\hat{X}_{t}=\sum_{n=1}^{N} q_{t}^{n} \tilde{s}_{t}^{n}$. For this application we use a bootstrap filter, i.e., the importance function $f$ is in fact the prior law $p\left(x_{t} \mid x_{t-1}\right)$. Each object produces one measurement at each time period according to (19) except during the time interval [600 700] where the first object does not produce any measurement and the second produces two $y^{1}$ and $y^{2}$ according to:

$$
Y_{t}=\arctan \left(\frac{x_{t}-x_{t}^{o b s}}{y_{t}-y_{t}^{o b s}}\right)+W_{t},
$$

where $W_{t}$ is a zero-mean Gaussian noise of covariance $\sigma_{w}^{2}$ independent of $V_{t} . x_{o b s}$ and $y_{o b s}$ are the Cartesian coordinates of the observer, which are known. The trajectories of the three targets and of the observer are plotted in figure 3.1 and the differences between the three couples of bearings simulated are plotted in figure 4 . As soon as the difference between two bearings issued from two different targets is lower than the standard deviation of the observation noise, the two measures cannot be distinguished, which makes this scenario very difficult. This difficulty is increased by the detection gap for the first object. One particular run of the particle filter with 5000 particles is presented in figure 3.2. The plot of the three estimated trajectories shows that the data association is overcome. There is no trajectories reversal and the estimations are quite satisfactory. Figure 5 shows the results of the estimation of the three components of $\pi_{t}$ and figure 3.3 represents the average of each component $\pi_{t}^{i}$ over successive intervals of 100 time steps and over the 20 trials. When there is an ambiguity about the origin of the measurements (i.e., when the differences between the bearings are lower than the standard deviation noise), the 

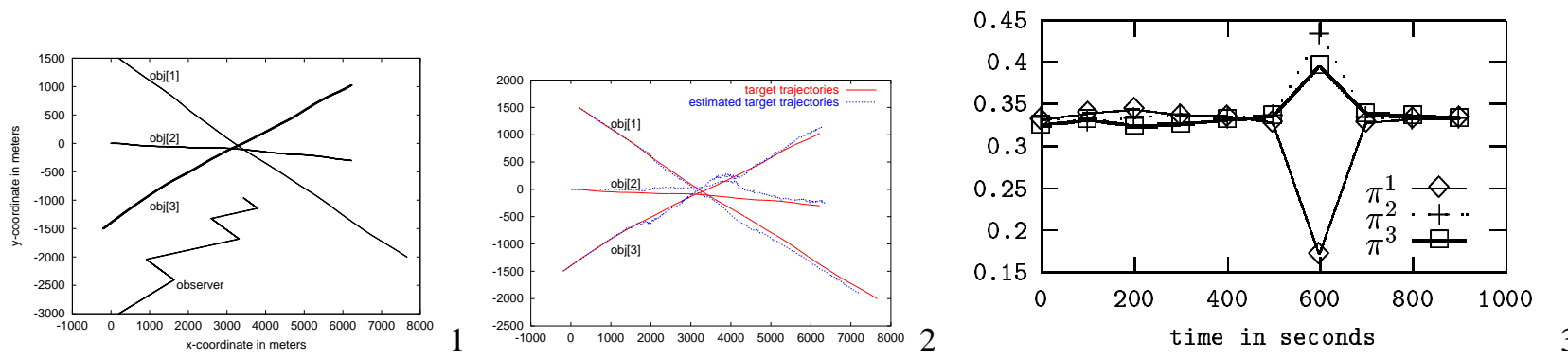

Figure 3. (1): Trajectories of the three targets and of the observer (2): The target trajectories and their estimation with 5000 particles (3): The average of the estimated components of the vector $\pi_{t}$ over the ten consecutive time intervals of length 100 and over 20 trials.
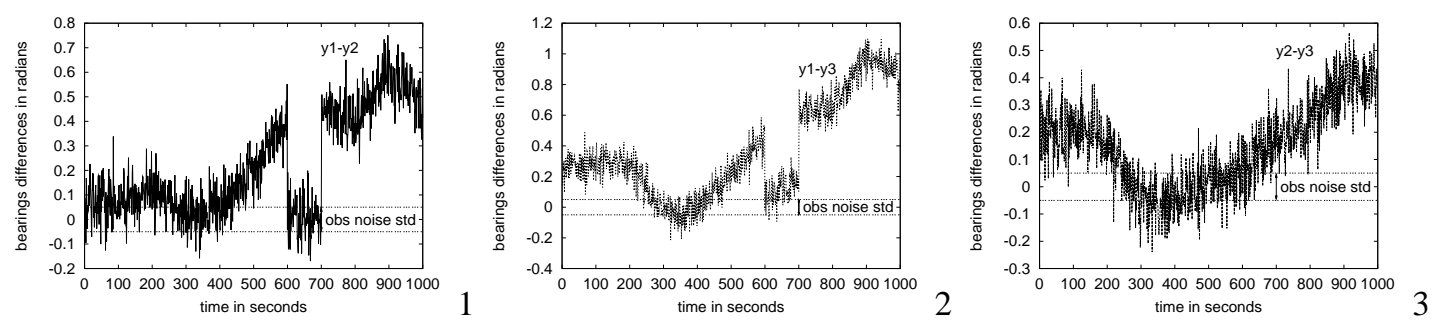

Figure 4. Differences between the three couples of target bearings at each time period compared to the standard deviation of the observation noise: (1) measurements 1 and 2 (2) 1 and 3 (3) 2 and 3.
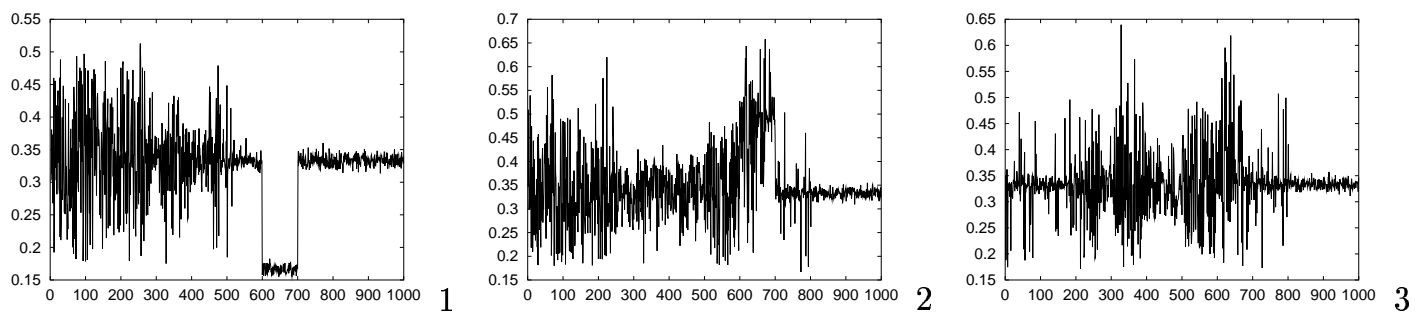

Figure 5. The estimated components of the vector $\pi_{t}$ obtained with 5000 particles: $(1) \hat{\pi}_{t}^{1},(2) \hat{\pi}_{t}^{2},(3) \hat{\pi}_{t}^{3}$.

components of $\pi$ vary in average around $1 / 3$ for $M=3$ objects and it stabilizes at uniform estimation $(1 / 3$ for $M=3$ objects) when the ambiguity disappears. The momentary measurement gap for the first object is correctly handled as the first component $\pi_{t}^{1}$ is instantaneously estimated as 0.15 from instant 600 to 700 .

The vector $\pi_{t}$ can then in turn help the estimation: an object leaving the surveillance or vision area can be detected by a drop of its $\pi_{t}$ component. On the other hand, the appearing of a new object might be related to an observation whose likelihood is low whatever object it is associated with. The statistic framework of our algorithm, through an interaction between the estimation of the objects trajectories and of the data association, seems then very promising in order to deal with the appearing and disappearing of objects of the study area, i.e. to deal with a varying number of objects.

\section{Application in image-based tracking}

We focus on a video sequence where three persons are moving according to unknown dynamics. As they are crossing in the 2D-plane of the images, the tracker must solve partial and complete occlusions as shown on figures $6 .\{1,2,3\}$. The modelization of the tracking problem consists in defining a state vector, its evolution model and an observation model. Such a design stage is quite easy in signal processing because the models and the different measures are well defined in the literature. It is not the case for the problem of video-based tracking: we have usually few knowledge on the a priori motion of the objects and any 

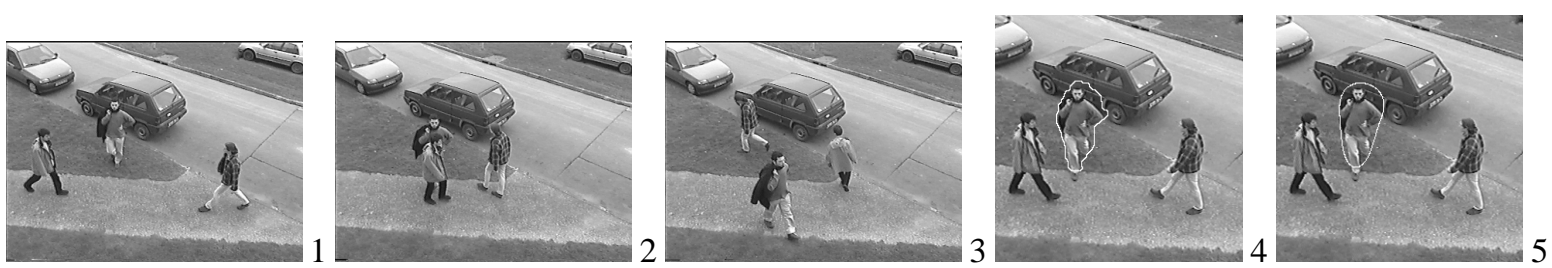

Figure 6. $(1,2,3)$ : Frames 7,30 and 67 of a pedestrian video-sequence with occlusions; (4): Outline of the motion area detected around one of the moving person; (5): Fourier contour obtained by inverse Fourier transform of the truncated discrete Fourier transform to the five first coefficients of the previous outline.
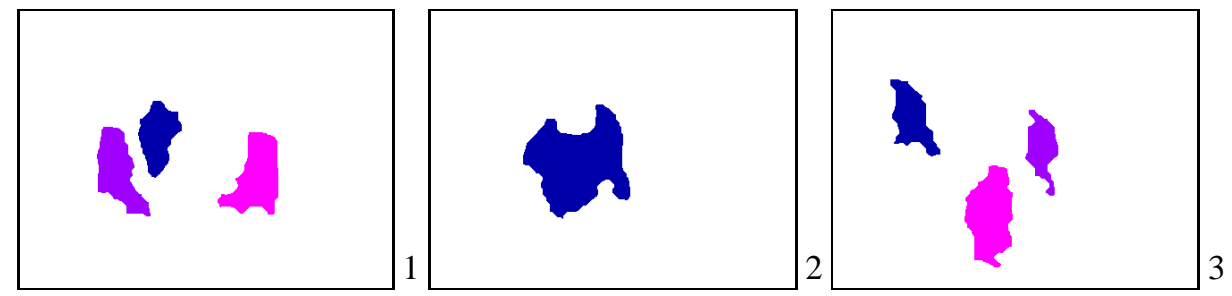

Figure 7. Maps of motion-based segmentation associated to the frames 19, 39 and 79.
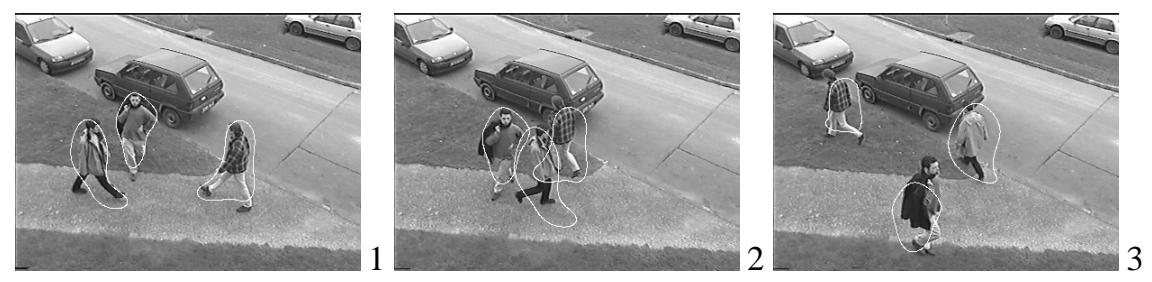

Figure 8. Estimated outlines of the three pedestrians with 100 particles and 9 Fourier coefficients for the frames 19,39 and 79 .

computation on the image can constitute a measurement.

\subsection{State space model}

In order to keep reasonable dimension of the state space, one have to come up with a compact representation of the object silhouette unless strong prior on the shape of the object of interest is available. An appealing and generic approach consists in using Fourier descriptors obtained by inverse Fourier transform of the truncated discrete Fourier transform of the initial object contour. For instance, the outline of one moving pedestrian (as observed through a motion segmentation map, see section 5.2) associated to the five first Fourier coefficients is presented in figure 6.5. For each object, the state vector is then composed of $2 N_{U}+1$ Fourier coefficients $X c_{t}^{i}$ and of the $2 \mathrm{D}$ translation vector $X v_{t}^{i}$ of the object center between $t-\Delta t$ and $t$ :

$$
X_{t}^{i}=\left(X c_{t}^{i}, X v_{t}^{i}\right)
$$

Notice that the object center is captured by the first Fourier coefficient denoted $X c_{t}^{i, 0}$. We use the following dynamics prior:

$$
\left\{\begin{array}{l}
X v_{t}^{i}=W_{t}^{i} \\
X c_{t}^{i, 0}=X c_{t-\Delta t}^{i, 0}+\Delta t X v_{t}^{i}+V_{t}^{i, 0} \\
X c_{t}^{i, k}=X c_{t-\Delta t}^{i, k}+V_{t}^{i, k} \text { for } k=-N_{U}, \ldots,-1,+1, \ldots, N_{U}
\end{array}\right.
$$

where $V_{t}$ is a Gaussian zero-mean noise of standard deviation $\sigma_{v}$ and $W_{t}$ is uniform on $\left[-V_{\max } ; V_{\max }\right]$.

\subsection{Measurements}

We use two types of measurements related to position and velocity of moving objects in the scene. By a motionbased segmentation of the image (see [8]), we first compute connected components of motion $C c_{t}^{j}$ for $j=1, \ldots, m_{t}$ as shown in figure 7 . Such a segmentation provides information on the localization (and shape) of the objects in motion 
w.r.t. the camera. The likelihood of the connected component $C c_{t}^{j}$ conditionnaly to the $i^{t h}$ component of the $n^{t h}$ particle $S c_{t}^{n, i}$ is chosen as:

$$
\frac{\left|\stackrel{\circ}{S} c_{t}^{n, i} \cap C c_{t}^{j}\right|}{\sqrt{\left|\stackrel{\circ}{S} c_{t}^{n, i}\right|}}
$$

where $\stackrel{\circ}{S} c$ denotes the inside of the closed contour defined by Fourier descriptors $S c$, and |.| stands for set cardinal. The denominator prevents a bias toward large contours. We then estimate the translation in the 2D-plane of each connected component between the frame at current time and the previous one. These translations will be noted $T_{t}^{j}$ for $j=1, \ldots, m_{t}$ and are supposed to arise from the model:

$$
T_{t}^{j}=X v_{t}^{i}+Z_{t}^{j} \text { if } K_{t}^{j}=i
$$

where $Z_{t}^{j}$ is a zero-mean Gaussian noise of std $\sigma_{z}$. As the velocity prior is very weak (uniform distribution, in (21)), we use these motion measurements to construct an importance function that will perform better than standard bootstrap-type choice based only on the dynamics, to guide particles toward regions of high likelihood.

\subsection{Importance function}

Given a partcile $s$, a translation estimation is considered available when only one of the object centers belongs to the connected component under consideration. Otherwise it means that several objects are observed through the same motion region (as in figure 7.2) and its global motion results in general from an intricate mix of individual motions. Then, if $s_{t-1}^{n, i}$ is the only object from $s_{t-1}^{n}$ whose center lies in $C c_{t}^{j}$, we propose the displacement of the $i$-th object in the new particle according to:

$$
\tilde{S} v_{t}^{n, i}=T_{t}^{j}+Z_{t}^{n, i}
$$

where $Z_{t}^{i}$ is the same noise as in (23). The other components $\tilde{S} c_{t}^{n, i}$ are predicted according to the dynamic prior in (21).

If no translation motion estimation is available, the new particle is displaced according to:

$$
\tilde{S} v_{t}^{n, i}=S v_{t-1}^{n, i}+Z_{t}^{n, i}
$$

\subsection{Importance weights}

The weight of the $n^{t h}$ particle is proportionnal to

$$
q_{t-1}^{n} \frac{p\left(\tilde{S} c_{t}^{n} \mid S_{t-\Delta t}^{n}\right) p\left(\tilde{S} v_{t}^{n} \mid S_{t-\Delta t}^{n}\right) p\left(T_{t} \mid \tilde{S}_{t}^{n}\right) p\left(C c_{t} \mid \tilde{S}_{t}^{n}\right)}{p\left(\tilde{S} c_{t}^{n} \mid S_{t-\Delta t}^{n}\right) f\left(\tilde{S} v_{t}^{n} \mid T_{t}\right)}
$$

As the prior on the motion is uniform, it does not affect the weights. When we use the current translation estimation, the choice of the importance function implies that $p\left(T_{t} \mid X_{t}\right)=p\left(T_{t} \mid X v_{t}\right)$ is equal to $f\left(X v_{t} \mid T_{t}\right)$. If this is the case for all the objects of the predicted particle, the new weight is obtained by multiplying the previous one by the likelihood of the connected component likelihood of the motion-based segmentation.

Result samples on multiple people tracking are presented in figure 8 .

\section{Conclusion}

We proposed multiple object tracking based on a mix of particle filtering and Gibbs sampling. Target state vectors and association probabilities are estimated jointly without enumeration, pruning or gating, by means of particle sets representing the joint a posteriori law of the target states. We have demonstrated the relevance of the approach both in bearings-only tracking and image-based tracking. We are currently investigating its extension to a varying number of objects, using parameters statistics.

\section{References}

[1] J. Diebolt and C. P. Robert. Estimation of finite mixture distributions through Bayesian sampling. Journal of the Royal Statistical Society series B, 56:363-375, 1994.

[2] A. Doucet. On sequential simulation-based methods for Bayesian filtering. Technical report, CUED/F-INFENG/TR 310, Signal Processing Group, Departement of Enginerring, University of Cambridge, 1998.

[3] T. E. Fortmann, Y. Bar-Shalom, and M. Scheffe. Sonar tracking of multiple targets using joint probabilistic data association. IEEE Journal of Oceanic Engineering, 8:173184, July 1983.

[4] N. Gordon, D. Salmond, and A. Smith. Novel approach to nonlinear/non-Gaussian Bayesian state estimation. IEE Proc.F, Radar and signal procesing, 140(2):107-113, 1993.

[5] M. Isard and A. Blake. CONDENSATION - conditional density propagation for visual tracking. Int. J. Computer Vision, 29(1):5-28, 1998.

[6] A. Kong, J. Liu, and W. Wong. Sequential imputation method and Bayesian missing data problems. J. Amer. Statist. Assoc., 89:278-288, 1994.

[7] J. MacCormick and A. Blake. A probabilistic exclusion principle for tracking multiple objects. In Proc. Int. Conf. Computer Vision, pages 572-578, 1999.

[8] J.-M. Odobez and P. Bouthémy. Direct incremental modelbased image motion segmentation for video analysis. Signal Processing, 66(2):143-155, Apr. 1998.

[9] M. Stephens. Bayesian Methods for Mixtures of Normal Distributions. PhD thesis, Magdalen College, Oxford, 1997.

[10] R. L. Streit and T. E. Luginbuhl. Maximum likelihood method for probabilistic multi-hypothesis tracking. In Proceedings of SPIE International Symposium, Signal and Data Processing of Small Targets 1994, volume 2335, 1994. 